\title{
Clark's Technique of Vestibuloplasty - A Case Report
}

\author{
Dr. Benju Shrestha, ${ }^{1}$ Dr. Krishna Prasad Lamichhane, ${ }^{1}$ Dr. Shaili Pradhan, ${ }^{1}$ \\ Dr Ranjita Shrestha Gorkhali, ${ }^{1}$ Dr. Pramod Kumar Koirala ${ }^{1}$ \\ ${ }^{1}$ Periodontology and Oral Implantology Unit, Department of Dental Surgery, \\ National Academy of Medical Sciences, Bir Hospital, Kathmandu, Nepal.
}

\begin{abstract}
Shallow vestibule can create a barrier in oral hygiene maintenance and can cause gingival recession due to the muscular traction. Inadequate vestibular depth in combination with inadequate attached gingiva is said to cause more food accumulation during mastication. Hence, shallow vestibule impeding with oral hygiene maintenance requires correction. Vestibuloplasty involves surgical procedure for repositioning of mucosa and muscle attachment thereby increasing the vestibular depth. There are various techniques of vestibuloplasty. This case report highlights upon the Clark's technique of vestibuloplasty for correction of shallow vestibule.

Keywords: Clark's technique; shallow vestibule; vestibuloplasty.
\end{abstract}

\section{INTRODUCTION}

Vestibular depth is measured either from crest of lip or from coronal border of the attached gingiva to depth of mucobuccal fold. ${ }^{1}$ Adequate vestibular depth is generally considered as vestibular depth that enables to maintain proper oral hygiene. ${ }^{2}$ Shallow vestibule often favours food accumulation and impedes oral hygiene maintenance. If combined with inadequate attached gingiva, it can cause pulling of marginal tissue and gingival recession. ${ }^{3}$ Vestibuloplasty procedure helps to deepen the vestibule by modifying soft tissue attachment. Different vestibuloplasty procedures are found to have varying degree of success. ${ }^{4}$ This case report describes Clark's technique ${ }^{5}$ of vestibuloplasty for managing shallow vestibule.

\section{CASE REPORT}

A 40 years old male presented to Periodontology and Oral Implantology Unit in Department of Dental Surgery, Bir hospital, National Academy of Medical Sciences (NAMS), Kathmandu, Nepal with the chief complaint of difficulty in brushing teeth on the lower left back region for eight months.

\section{Correspondence:}

Dr. Benju Shrestha

Periodontology and Oral Implantology Unit, Department of Dental Surgery, National Academy of Medical Sciences, Bir Hospital, Kathmandu, Nepal.

email: benjushrsth539@gmail.com

\section{Citation}

Shrestha B, Lamichhane KP, Pradhan S, Gorkhali RS, Koirala PK. Clark's Technique of Vestibuloplasty. J Nepal Soc Perio Oral 93-5
The patient had tobacco chewing habit and developed white lesion in lower left back region. He was advised for cessation of tobacco chewing but the lesion did not resolve even after habit cessation. Excisional biopsy was then performed. Following healing of excisional wound the patient had difficulty in brushing in lower left back region.

On clinical examination, the patient had generalised gingival recession and reduced vestibular depth with respect to 44 to 47 . The muscle attachment was almost near the gingival margin (Figure 1) in mandibular left posterior region. Intraoral periapical radiograph showed horizontal bone loss with respect to 45,46 , and 47 within cervical third of the root length (Figure 2). The clinical probing depths were normal. After four weeks of non-surgical periodontal therapy, vestibuloplasty (Clark's technique) was performed.

Procedure: Under local anaesthesia, horizontal incision was placed at mucogingival junction with respect to $44,45,46$ and 47 which was followed by supraperiosteal dissection up to the desired vestibular depth (Figure 3). The mucosa on the buccal side was then undermined and the mucosal flap was sutured with resorbable synthetic vicryl 4-0 suture at the depth of vestibule (Figure 4). The raw area over the alveolar bone was left to heal by secondary intention. Coepack periodontal dressing was placed for wound protection (Figure 5) which was removed after 10 days. The healing after two weeks is shown in Figure 6 where increased vestibular depth can be appreciated. After three months (Figure 7), there was reduction in vestibular depth compared to two weeks. However, there was adequate attached gingiva and the patient could maintain his oral hygiene. 


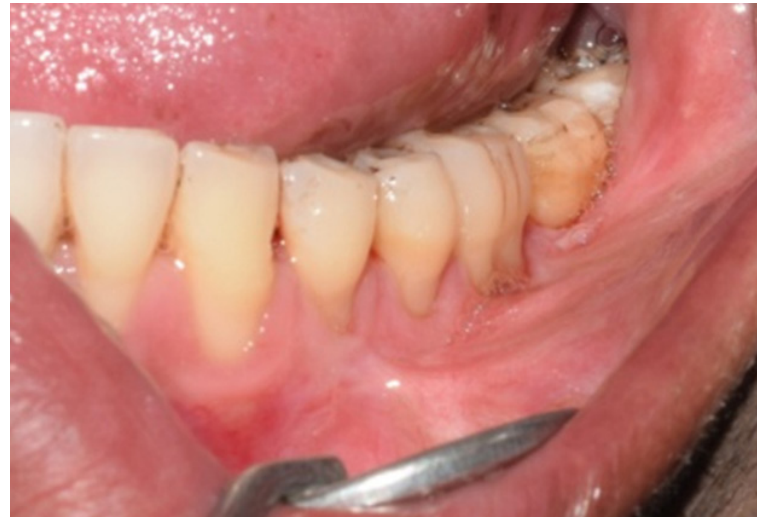

Figure 1: Shallow buccal vestibule with inadequate attached gingiva in left posterior mandibular region.

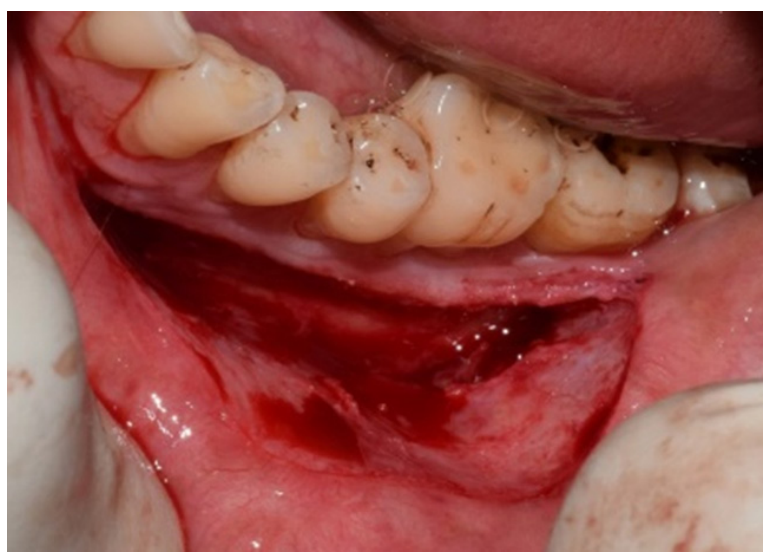

Figure 3: Horizontal incision placed at mucogingival junction followed by supraperiosteal dissection.

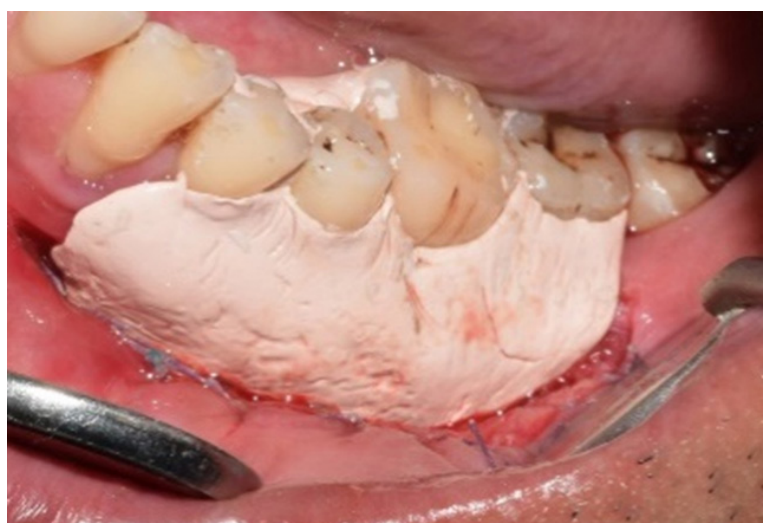

Figure 5: Coe-pack placed over raw areas on alveolar bone.

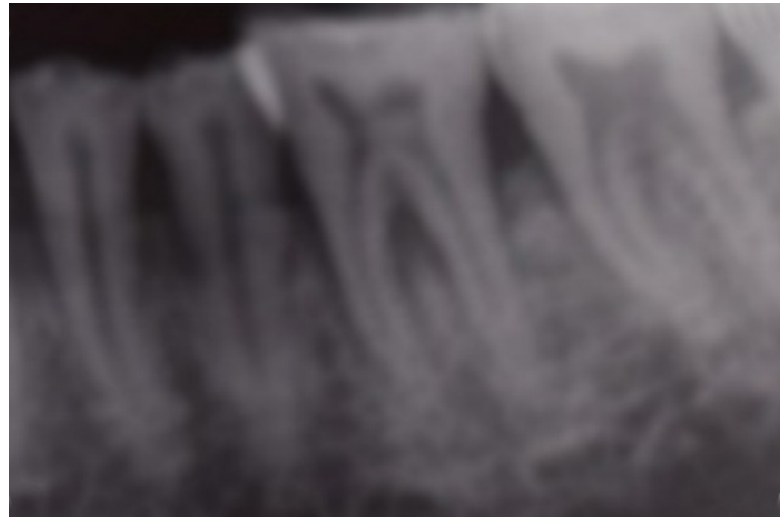

Figure 2: Intraoral periapical radiograph with respect to 44 to 47 .

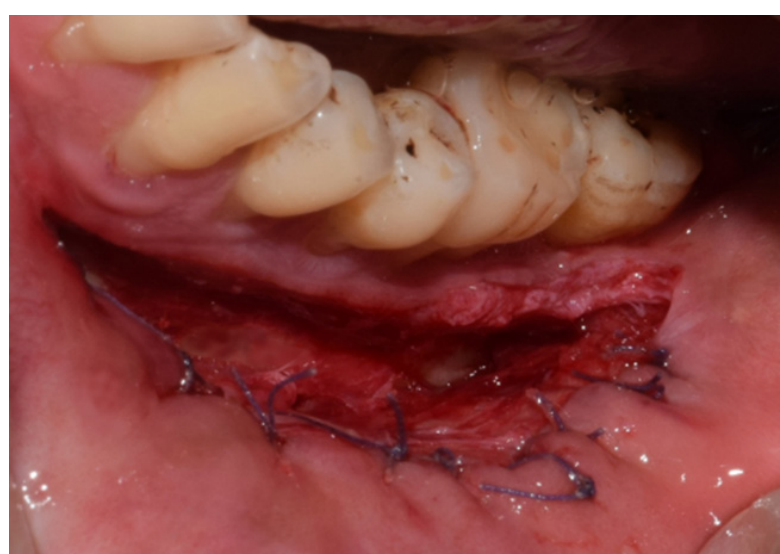

Figure 4: Mucosal flap sutured to the depth of vestibule.

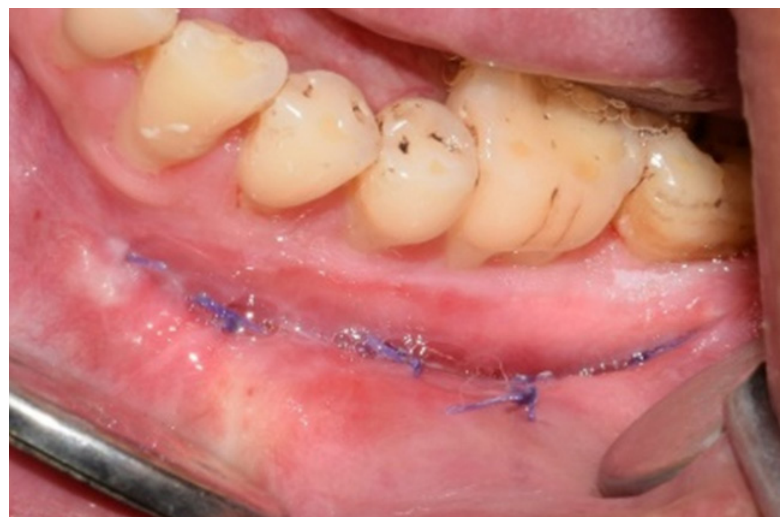

Figure 6: After two weeks of vestibuloplasty.

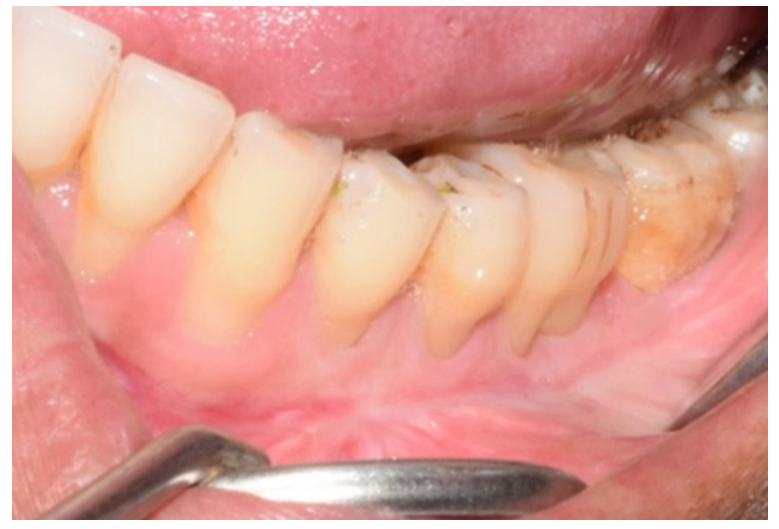

Figure 7: After three months of vestibuloplasty. 


\section{DISCUSSION}

Freidman (1957) described the term "mucogingival surgery" as a surgical procedure that corrects the relationship between the gingiva and oral mucosa with reference to three areas - attached gingiva, shallow vestibule, and aberrant frenum that interfere in the maintenance of marginal gingival health. ${ }^{6}$ Shallow vestibule can occur as a result of alveolar ridge atrophy after extraction, high muscle insertion or as a result of surgical procedures such as coronally advanced flaps, excisional wound followed by scarring, etc. Shallow vestibule has been associated with problems such as barrier in maintenance of oral hygiene, increased food accumulation during mastication, gingival recession, difficulty in placing removable prosthesis, etc. ${ }^{3,4}$ Hence, correction of shallow vestibule is needed to maintain the periodontal health. In the present case, the patient had difficulty in brushing due to shallow vestibule which was corrected by vestibuloplasty.

Vestibuloplasty is the surgical modification of the gingivamucous membrane relationships including deepening of the vestibular trough, altering the position of the frenulum or muscle attachments, and widening of the zone of attached gingiva. ${ }^{7}$ There are various types of vestibuloplasty namely mucosal advancement vestibuloplasty, secondary epithelisation vestibuloplasty and grafting vestibuloplasty. ${ }^{4}$ In mucosal advancement vestibuloplasty, the mucous membrane of the vestibule is undermined and advanced to line both sides of the extended vestibule while in secondary epithelisation vestibuloplasty, one side of the extended vestibule is left to heal by secondary intention. In grafting vestibuloplasty, dermal or mucosal grafts can be used to line one or both sides of the extended vestibule.

Adequate mucosal length must be available for mucosal advancement vestibuloplasty. If a tongue blade or mouth mirror is placed to the height of the vestibule without distortion or inversion of the lip, adequate mucosa is present for mucosal advancement vestibuloplasty. But if distortion occurs then, secondary epithelisation vestibuloplasty is the appropriate procedure. ${ }^{5,8}$ In current case report, mucosa was not adequate hence, secondary epithelisation vestibuloplasty was preferred.

Kazanjian's technique (1924) is the prototype of vestibuloplasty procedure where the labial flap pedicled off the alveolar process is used to cover the alveolar bone side while the labial surface is allowed to heal by secondary epithelisation. ${ }^{9}$ The major drawback of this procedure is severe scarring of lip causing reduced lip flexibility. Thus, to overcome this drawback Clark (1953) recommended flap to be pedicled off the lip and raw area was left on the alveolar side rather than labial side. ${ }^{9}$

The disadvantages of Clark's technique is there can be unpredictable amount of relapse of the vestibular depth gained and scarring of vestibule. ${ }^{9}$ The secondary epithelisation vestibuloplasty heals similar as excision wound. Hence, during healing, the newly attached mucosa becomes loose and the detached muscles reinsert into preoperative levels causing relapse. ${ }^{10}$ Reduction in the vestibular depth can be seen over three months follow-up in current case report. However, with the residual vestibular depth, patient could perform oral hygiene procedures comfortably than compared to baseline. It is suggested that the problem of relapse can be overcome by over correction or use of skin or mucosal grafts over denuded area.

\section{Conflict of Interest: None.}

\section{REFERENCES}

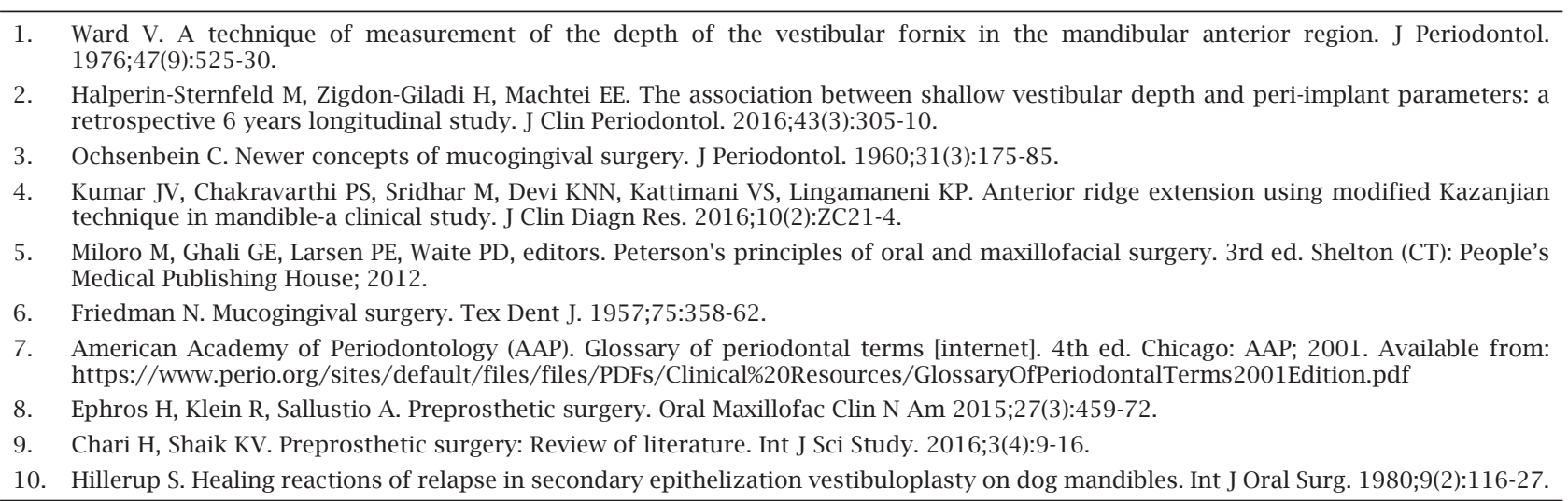

